\title{
Erratum to: Exploring motor and visual imagery in Amyotrophic Lateral Sclerosis
}

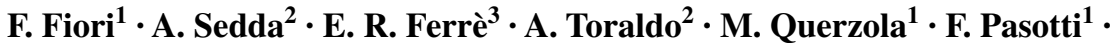

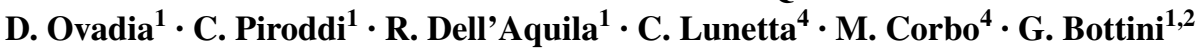

Published online: 2 March 2016

(C) Springer-Verlag Berlin Heidelberg 2016

\section{Erratum to: Exp Brain Res (2013) 226:537-547 DOI 10.1007/s00221-013-3465-9}

Upon review, the authors discovered errors in the values given in Table 2. Table 2, as corrected, should read as follows:

Table 2 Average percentage of correct answers for control group and ALS patients

\begin{tabular}{|c|c|c|c|c|c|c|c|c|}
\hline \multirow{3}{*}{$\begin{array}{l}\text { Orien- } \\
\text { tations }\end{array}$} & \multicolumn{4}{|l|}{ HLT } & \multicolumn{4}{|l|}{ MLD } \\
\hline & \multicolumn{2}{|c|}{ Controls } & \multicolumn{2}{|c|}{ ALS patients } & \multicolumn{2}{|c|}{ Controls } & \multicolumn{2}{|c|}{ ALS patients } \\
\hline & Mean & SE & Mean & SE & Mean & SE & Mean & SE \\
\hline $0^{\circ}$ & 95.38 & \pm 1.44 & 92.90 & \pm 1.46 & 95.83 & \pm 1.30 & 88.59 & \pm 2.31 \\
\hline $90^{\circ}$ & 84.96 & \pm 2.86 & 86.99 & \pm 1.46 & 91.49 & \pm 1.86 & 84.12 & \pm 2.27 \\
\hline $180^{\circ}$ & 83.06 & \pm 2.54 & 76.78 & \pm 1.38 & 79.08 & \pm 3.62 & 74.88 & \pm 2.56 \\
\hline $270^{\circ}$ & 96.74 & \pm 0.55 & 83.21 & \pm 1.46 & 92.66 & \pm 1.59 & 83.27 & \pm 1.84 \\
\hline
\end{tabular}

Patients' and controls' average percentage of correct answers ( \pm standard error $[\mathrm{SE}]$ of the mean) as a function of stimulus orientation (collapsed between palm/dorsum view and left/right laterality [hands] and $\mathrm{F} / \mathrm{J}$ and canonical/mirror-reversed view [letters]). For the HLT, we adopted Parsons (1987) original distinction between stimuli across the body midline and stimuli away from the body midline to estimate the accuracies for $90^{\circ}$ and $270^{\circ}$ rotated hands stimuli (see main text for details)

The online version of the original article can be found under doi:10.1007/s00221-013-3465-9.

G. Bottini

g.bottini@unipv.it

1 Center of Cognitive Neuropsychology, A.O. Niguarda Ca' Granda, Piazza Ospedale Maggiore no. 3, 20162 Milan, MI, Italy

2 Department of Brain and Behavioural Sciences, University of Pavia, Piazza Botta no. 11, 27100 Pavia, PV, Italy

3 Institute of Cognitive Neuroscience (ICN), University College London, Alexandra House, 17 Queen Square, London WC1N 3AR, UK

4 Neuromuscolar Omnicenter (NEMO), A.O. Niguarda Ca' Granda, Piazza Ospedale Maggiore no. 3, 20162 Milan, MI, Italy
Moreover, the authors also discovered three (3) typos in the reported Results (mixed ANOVA) on the hand laterality task (HLT): The paper reports 113.484 for the Angle of rotation by Stimulus view interaction, where the degrees of freedom should actually be 80.466 . Secondly, the $F$ for the factor Angle of rotation should be 33.669 and not 36.670. Finally, the $t$ value of the Effect of stimulus orientation contains an inversion in digits: It should read 4.629 and not 4.692 . 